\title{
Bacteriophage EK99P-1 Alleviates Enterotoxigenic Escherichia Coli K99-Induced Barrier Dysfunction and Inflammation
}

\section{Narae Kim}

Seoul National University

Min jeong Gu

Seoul National University

Yoon-Chul Kye

Seoul National University

Young-Jun Ju

Seoul National University

\section{Rira Hong}

Seoul National University

Do Bin Ju

Seoul National University

Young Jin Pyung

Seoul National University

\section{Seung Hyun Han}

Seoul National University

Byung-Chul Park

Seoul National University

Cheol-Heui Yun ( $\nabla$ cyun@snu.ac.kr)

Seoul National University

\section{Research Article}

Keywords: Bacteriophage EK99P-1, enterotoxigenic, Escherichia, barrier dysfunction, inflammation, antibiotics, immune responses

Posted Date: October 13th, 2021

DOI: https://doi.org/10.21203/rs.3.rs-850419/v2

License: (a) (i) This work is licensed under a Creative Commons Attribution 4.0 International License. Read Full License 
Version of Record: A version of this preprint was published at Scientific Reports on January 18th, 2022. See the published version at https://doi.org/10.1038/s41598-022-04861-4. 


\section{Abstract}

Bacteriophages have long been used as a potential alternative to antibiotics for livestock due to their ability to specifically kill ETEC, which are a major cause of diarrhea in piglets. However, the control of ETEC infection by phages within intestinal epithelial cells, and their relationship with host immune responses, remain poorly understood. In this study, we evaluated the effect of phage EK99P-1 against ETEC K99-infected IPEC-J2. Phage EK99P-1 prevented ETEC K99-induced barrier disruption by attenuating the increased permeability mediated by the loss of tight junction proteins such as ZO-1, occludin, and claudin-3. ETEC K99-induced inflammatory responses, such as IL-8 secretion, were decreased by treatment with phage EK99P-1. We used a IPEC-J2/PBMC Transwell co-culture system to investigate whether the modulation of barrier disruption and chemokine secretion by phage EK99P-1 in ETEC K99-infected IPEC-J2 would influence basolateral immune cells. The results showed that phage EK99P-1 reduced the mRNA expression of ETEC K99-induced pro-inflammatory cytokines, IL-1 $\beta$ and $I L-8$, from PBMC collected on the basolateral side. Together, these results suggest that phage EK99P-1 prevented ETEC K99-induced barrier dysfunction in IPEC-J2 and alleviated inflammation caused by ETEC K99 infection. Reinforcement of the intestinal barrier by phage EK99P-1 also modulates the immune cell inflammatory response.

\section{Introduction}

ETEC-associated diarrhea is an economically important disease $[1,2]$ that causes high morbidity and mortality in neonatal and weaned piglets $[3,4]$. Most, if not all, ETECs adhere to small intestinal epithelial cells (IECs) via fimbriae-mediated adhesion [5], as an initial step in infection [6]. Once they enter the host cell, enterotoxins like heat-labile toxin (LT) or heat-stable toxin (ST) are secreted, increasing the paracellular permeability of the host [7, 8]. IPEC-J2, originating from the small intestine jejunum of pigs, is used as a model for studying interactions between intestinal cells and enteric bacteria, including ETEC [9].

Previous studies have shown that ETEC-infected IPEC-J2 exhibit decreased expression of tight junction proteins such as occludin, claudin, and ZO-1, coincident with the secretion of chemokines such as monocyte chemoattractant protein(MCP)-1 and IL-8, which recruit immune cells into the local inflammatory site [10]. Pro-inflammatory cytokines, including IL-1 $\beta$, IL-2, IL-6, IL-12p40, interferon(IFN)- $\gamma$, and tumor necrosis factor(TNF)-a, have been observed in serum from ETEC-infected pigs [11].

Diarrheal diseases caused by ETEC in the pig industry can be prevented and treated using antibiotics [12, 13]; however, concerns over the increasing emergence of antibiotic-resistant bacteria together with global bans of antibiotics as feed additives have prompted efforts to develop alternatives to antibiotics [14], including lytic bacteriophages and their lysines.

Bacteriophages have long been used as an alternative to antibiotics to treat bacterial infection [15]. Infeed phages have been shown to reduce the severity of ETEC-induced diarrhea in newborn [16] and postweaning $[17,18]$ piglets; they have also been shown to alleviate symptoms such as increased rectal 
temperature and E. coli adhesion concordant with reducing serum IL-8 and TNF-a [12]. However, limited information is available on the effect of phages on IECs in terms of regulating host immune responses upon ETEC infection.

Therefore, in this study, we investigated the beneficial effects of phage EK99P-1 on barrier protection in IECs, and the inflammatory response involving mucosal immune cells, under ETEC K99 infection.

\section{Materials And Methods}

The use of porcine blood and experimental protocols of the present study were approved by the Institutional Animal Care and Use Committee of Seoul National University and all methods were performed in accordance with the relevant guidelines and regulations.

\section{Cell culture}

IPEC-J2 cells (DSMZ, Germany) were cultured with Dulbecco's modified Eagle's medium and F12 Ham's medium (DMEM-F12; Gibco, USA) containing 5\% heat-inactivated fetal bovine serum (FBS; Corning, USA), $1 \%$ insulin-transferrin-selenium-X (ITS-X; Gibco), and $1 \%$ antibiotics in an incubator at an atmosphere of $5 \% \mathrm{CO}_{2}$ and $39^{\circ} \mathrm{C}$. For differentiation, IPEC-J2 cells were seeded at $1 \times 10^{5}$ cells $/ \mathrm{mL}$ in $500 \mu \mathrm{L}$ DMEM medium, as described above, on $1.12-\mathrm{cm}^{2}$ polyester membrane inserts (pore size, $0.4 \mu \mathrm{m}$ ); the basolateral side was filled with $1 \mathrm{~mL}$ DMEM. After 4 days, the culture medium was replaced with differentiation medium (DMEM-F12 supplemented with $5 \%$ heat-inactivated FBS and $1 \%$ antibiotics), cultured for $7-10$ days, and differentiated within the insert until they attained a transepithelial electrical resistance (TEER) of $>5,000 \Omega$.

\section{Preparation of ETEC K99 and phage EK99P-1}

ETEC K99 and phage EK99P-1 [29] were donated by iNtRON Biotechnology (Korea). ETEC K99 was spread on an agar plate containing $3 \%$ tryptic soy broth (TSB), 1.5\% Bacto Agar (all from BD Biosciences, USA) and incubated at $37^{\circ} \mathrm{C}$ overnight. One colony was selected for inoculation in $10 \mathrm{~mL} 3 \% \mathrm{TSB}$ media, and incubated for $4-5 \mathrm{~h}$ in a shaking incubator at $150 \mathrm{rpm}$ and $37^{\circ} \mathrm{C}$ until the optical density (OD) reached 1.0. Then, phage EK99P-1 was diluted in elution buffer (10 mM Tris-Cl, pH 8.5) at a density of 7.3 $\times 10^{8}$ plaque-forming units $(\mathrm{pfu}) / \mathrm{mL}$.

\section{Bacterial infection and adhesion assay}

Differentiated IPEC-J2 cells cultured in a Transwell were washed with pre-warmed phosphate-buffered saline (PBS) and then placed in infection media (DMEM-F12 supplemented with $5 \%$ heat-inactivated FBS without antibiotics). The cells were infected with ETEC K99 at a density of $1 \times 10^{7} \mathrm{cfu} / \mathrm{mL}$ for $24 \mathrm{~h}$ at $37^{\circ} \mathrm{C}$ and $5 \% \mathrm{CO}_{2}$. For the adhesion assay, infected IPEC-J2 cells were washed with pre-warmed PBS three times and then lysed in $0.1 \%$ Triton X-100 for 5 min. The lysates were serial diluted and each inoculum, 
plated onto TSB agar and stored overnight. Viable bacterial cells were quantified as colony-forming units (cfu).

\section{TEER measurement}

IPEC-J2 cells were grown on a $1.12-\mathrm{cm}^{2}$ polyethylene terephthalate membrane insert (pore size, 0.4; Corning, USA) and treated with ETEC K99 and/or phage EK99P-1. TEER was measured using an epithelial volt/ohm meter (EVOM2; World Precision Instruments, USA). Briefly, 0.5 and $1.0 \mathrm{~mL}$ of pre-equilibrated medium were added to the apical and basal chambers. Measurements were performed after obtaining a steady signal for $5 \mathrm{~min}$ in the blank insert, and corrected by subtracting the background of the blank Transwell inserts and medium-only inserts. The final TEER reading was reported in $\Omega \mathrm{cm}^{2}$ (TEER measurement $\times$ area of membrane).

\section{Dextran permeability measurement}

Differentiated IPEC-J2 cells were cultured in a Transwell and treated with ETEC K99 and phage EK99P1 for $24 \mathrm{~h}$, and then washed with pre-warmed PBS three times. Cell culture medium containing 4 or 40 $\mathrm{kDa}$ dextran conjugated with fluorescein isothiocyanate (dextran-FITC) was added to the upper compartment of the Transwell plate at $2.2 \mathrm{mg} / \mathrm{mL}$. After $1 \mathrm{~h}$ of incubation, the fluorescence intensity in the lower compartment of the Transwell plate was measured using a fluorescence multiple plate reader (Victor 3; Perkin Elmer, USA). The excitation and emission wavelengths were 490 and $520 \mathrm{~nm}$, respectively.

\section{Porcine peripheral blood mononuclear cell (pPBMC) isolation}

Porcine blood samples were obtained from 4- to 6-month-old Landrace-Yorkshire-Duroc pigs (Hyupsin Food Co., Ltd., Korea). The use of porcine blood was approved by the Institutional Animal Care and Use Committee of Seoul National University (IACUC no. SNU-150327-2). Porcine whole blood was diluted with PBS at a ratio of 1:1, and pPBMCs were isolated by density gradient centrifugation ( $400 \times g$ for 20 min without brake) using Ficoll-Paque Plus (Amersham Bioscience, UK)[30]. pPBMCs were suspended in RPMI 1640 medium supplemented with $10 \%$ FBS and $1 \%$ penicillin/streptomycin.

\section{Co-culture IPEC-J2/PBMCs}

IPEC-J2 cells were seeded at a density of $1 \times 10^{5}$ cells $/ \mathrm{mL}$ in $500 \mu \mathrm{L}$ DMEM medium as described above on $1.12-\mathrm{cm}^{2}$ polyester membrane inserts (pore size, 0.4 ); the basolateral side was filled with $1 \mathrm{~mL}$ DMEM. During cell growth and differentiation, the medium in both compartments was replaced three times per week for 7-9 days. Then, pPBMCs $\left(2 \times 10^{6}\right.$ cells $\left./ \mathrm{mL}\right)$ were seeded in the basolateral compartment of the Transwell plate with $1 \mathrm{~mL}$ RPMI media, and ETEC K99 and phage EK99P-1 were added to the apical compartment.

\section{Western blot analysis}


To examine the effect of phage EK99P-1 on tight junction proteins, $1 \times 10^{5}$ cells were cultured on 12-well plates for 2 days until confluence was reached. Confluent IPEC-J2 cells were treated with ETEC K99 and/or phage EK99P-1, washed with PBS and then lysed in a RIPA lysis buffer (50 mM Tris- $\mathrm{HCl}, 150 \mathrm{mM}$ $\mathrm{NaCl}, 1 \%$ NP_40, $0.5 \%$ sodium deoxycholate, $0.1 \%$ sodium dodecyl sulfate containing protease inhibitor), followed by protein quantitation using a Micro BCA kit (Thermo, USA). The same amount of extract was loaded into $12 \%$ Tris-glycine polyacrylamide gel and electrophoresed. Then, the proteins were transferred onto a polyvinylidene fluoride microporous membrane for $90 \mathrm{~min}$ at $4^{\circ} \mathrm{C}$ and blocked with $5 \%$ skim milk in TBS-T (1 M Tris- $\mathrm{HCl}, 5 \mathrm{M} \mathrm{NaCl}, 10 \%$ Tween-20) for $1 \mathrm{~h}$. The blot was incubated with rabbit anti-claudin-3, occludin, and -ZO-1 or mouse anti- $\beta$-actin antibodies (Invitrogen, USA) overnight. Then, the membrane was washed and incubated with goat anti-rabbit or anti-mouse IgG-HRP antibody (Santa Cruz Biotechnology, USA) for $1 \mathrm{~h}$. The target protein was visualized using an enhanced chemiluminescence system (GE Healthcare, USA), followed by analysis using a ChemiDoc XRS system (Bio-Rad, USA).

\section{Real-time quantitative reverse transcription polymerase chain reaction (qRT-PCR)}

Total RNA was isolated using TRIzol reagent according to the manufacturer's instructions and reversetranscribed to generate complementary DNA (cDNA) using oligo-dT primers (Bioneer, Korea). Real-time qRT-PCR was performed using a StepOne Plus real-time PCR system (Applied Biosystems, USA). The PCR reaction was conducted in a 96-well reaction plate using $9 \mu \mathrm{L}$ SYBR green PCR Master Mix, $1 \mu \mathrm{L}$ each of forward and reverse primers, $1 \mu \mathrm{L} \mathrm{cDNA}$ template, and $8 \mu \mathrm{L}$ nuclease-free $\mathrm{H}_{2} \mathrm{O}$. The PCR conditions included 40 thermal cycles of $2 \mathrm{~min}$ at $50^{\circ} \mathrm{C}, 10 \mathrm{~min}$ at $95^{\circ} \mathrm{C}, 15 \mathrm{~s}$ at $95^{\circ} \mathrm{C}, 30 \mathrm{~s}$ at $60^{\circ} \mathrm{C}$, and $30 \mathrm{~s}$ at $72^{\circ} \mathrm{C}$. Relative quantification of the target genes was calculated using the $2^{-\triangle \Delta C t}$ method. Target gene expression was normalized to glyceraldehyde 3-phosphate dehydrogenase (GAPDH) or $\beta$-actin mRNA level. Primer sequences used in this experiment are shown in Table 1.

\section{Proportional changes of immune cells}

We washed pPBMCs with PBS containing 1\% FBS and performed staining using the following monoclonal antibodies at pre-determined optimal concentrations: mouse anti-porcine CD3e (clone PPT3; Southern Biotech, USA), CD4 FITC (clone 74-12-4; BD Biosciences), CD8a PE (clone 76-2-11; BD Biosciences), and CD172a biotin (clone 74-22-15; BD Biosciences). Rat anti-mouse IgG1 APC (clone A851, BD Biosciences) and streptavidin BV605 (Biolegend, USA) were used as the secondary antibody. The cells were incubated for $20 \mathrm{~min}$ at $4^{\circ} \mathrm{C}$ in the dark. After staining, the cells were washed and the expression of surface markers was measured using flow cytometry (FACSCanto Il; BD Biosciences). All flow cytometric data were analyzed using the FlowJo software (Tree Star, USA).

\section{Apoptosis analysis}

Floating cells were collected and the attached cells were trypsinized for 5 min and washed with PBS. Finally, both trypsinized and floating cells were added together and stained with Annexin V-APC and propidium iodide (PI). Marker intensity was examined using flow cytometry (FACSCanto II; BD Biosciences). All flow cytometry data were analyzed using the FlowJo software (Tree Star). 


\section{Statistical analyses}

Means \pm standard deviation were determined on the basis of at least three different samples. All experiments were performed at least three times. Means were compared between two groups using twotailed paired Student's $t$-tests. The groups were compared by one-way analysis of variance (ANOVA), followed by the Friedman test and Tukey's multiple comparison test. All statistical analyses were performed using GraphPad Prism software (v5.01; GraphPad Software, USA). Significance was evaluated at a level of $P<0.05$.

\section{Results}

\section{Phage EK99P-1 restrained intestinal barrier disruption}

ETEC induces increased intestinal permeability, resulting in reduced barrier protection [8]. To determine whether phage EK99P-1 treatment alleviates intestinal barrier function damage caused by ETEC K99 infection, we treated differentiated IPEC-J2 cells with either ETEC K99 or phage EK99P-1, or both, on the apical side. Decreased TEER values due to ETEC K99 infection were restored by phage EK99P-1 treatment in a dose-dependent manner (Figure 1A). IPEC-J2 cell death did not occur at any concentration of phage EK99P-1 in this experiment (Figure S1). TEER values of differentiated IPEC-J2 cells were maintained until $24 \mathrm{~h}$ after treatment with both ETEC K99 and phage EK99P-1, whereas treatment with ETEC K99 alone significantly reduced TEER (Figure 1B). This result suggests that phage EK99P-1 treatment minimizes damage and inhibits the increased permeability induced by ETEC K99.

Soluble toxins, such as LT and ST, are able to permeate the IEC barrier. The molecular weights of LT and $\mathrm{ST}$ are $\sim 80$ and $2 \mathrm{kD}$, respectively; they may migrate to the lamina propria across the epithelial layer according to their paracellular permeability [2]. To test this hypothesis, we used a dextran-FITC transepithelial permeability assay to quantify the effect of ETEC K99 and phage EK99P-1 on paracellular permeability: 4- or 40-kD dextran-FITC permeability was maintained in IPEC-J2 cells treated with ETEC K99 and phage EK99P-1, whereas ETEC K99 alone showed significant dextran permeability (Figure 1C, D). These results demonstrate that phage EK99P-1 efficiently prevented the increased intestinal permeability associated with ETEC K99 infection. No significant changes in permeability were observed upon treatment with phage EK99P-1 alone, because no target bacteria of phage EK99P-1 were present (Figure 1C, D). Together, these results show that phage EK99P-1 ameliorates the intestinal barrier disruption and permeability associated with ETEC K99 infection.

\section{Phage EK99P-1 inhibited the loss of tight junction integrity in IPEC-J2 cells infected with ETEC K99}

Our previous study showed that differentiated IPEC-J2 cells form a polarized monolayer with increased TEER values, concordant with increased expression of tight junction proteins such as ZO-1, occludin, and claudin-3 [19]. Because the increase in permeability is partly caused by reduced expression of these tight junction proteins, we examined the expression of ZO-1, occludin, and claudin-3 in IPEC-J2 cells infected with ETEC K99. Consistent with our permeability results (Figure 1), disrupted tight junction proteins were 
restored in ETEC K99-infected IPEC-J2 cells through phage EK99P-1 treatment (Figure 2). These results suggest that phage EK99P-1 treatment effectively prevents intestinal barrier disruption through inducing increased tight junction proteins in IPEC-J2 cells infected with ETEC K99.

\section{Phage EK99P-1 prevented ETEC K99 adherence to IPEC-J2 cells}

ETEC adhesion to IECs during infection plays an important role in inducing inflammatory responses and disrupting barrier function at the intestinal lining [8]. Therefore, we hypothesized that the restoration of permeability by phage EK99P-1 in ETEC K99-infected IPEC-J2 cells could be related to the initial adhesion of ETEC K99 to IPEC-J2 cells. To examine the interference of phage EK99P-1 in the attachment of ETEC K99 to IPEC-J2 cells, we measured the cfu of cell-adhesive ETEC K99. We found that phage EK99P-1 treatment reduced ETEC K99 adhesion to IPEC-J2 cells (Figure 3). We observed no ETEC K99 translocation from the apical to the basolateral side, with or without phage EK99P-1 (Figure S2). This result suggests that the intestinal barrier protection conferred by phage EK99P-1 treatment may have begun with reduced ETEC K99 adhesion to IPEC-J2 cells due to the interference of phage EK99P-1.

\section{Phage EK99P-1 alleviated inflammatory responses of IPEC-J2 cells infected with ETEC K99}

IECs have a barrier function that involves sensing and responding to microbial stimuli, and also participate in the coordination of inflammatory responses. For example, IECs secrete cytokines and chemokines upon recognition of ETEC adhesion [20] to alert the underlying mucosal immune cells [21]. To investigate whether phage EK99P-1 treatment modulates inflammatory responses following ETEC K99 infection, we examined the release of cytokines and chemokines in IPEC-J2 cells treated with ETEC K99 and phage EK99P-1. Compared to treatment with ETEC K99 alone, simultaneous treatment of ETEC K99 and phage EK99P-1 significantly reduced the mRNA expression of IL-8 and MCP-1 (Figure $4 \mathrm{~A}$ ), and significantly decreased IL-8 secretion (Figure 4B). These results suggest that phage EK99P-1 alleviates inflammatory responses in IPEC-J2 cells infected with ETEC K99.

\section{Phage EK99P-1 reduced inflammatory cytokines in PPBMC co-cultured with ETEC K99-infected IPEC-J2}

IECs are thought to participate in the recognition of bacterial components and transduce signals to resident mucosal immune cells [22]. To investigate the effect of phage EK99P-1 treatment on the modulation of underlying mucosal immune responses by IECs, differentiated IPEC-J2 cells were cocultured with pPBMCs using a previously established Transwell co-culture system [23]. We investigated the production of pro-inflammatory cytokines and chemokines, $I L-1 \beta, I L-8$, and $I F N-\gamma$, in co-cultured pPBMCs. The mRNA expression levels of $I L-1 \beta, I L-8$,and $I F N-\gamma$ were significantly increased under ETEC K99 alone compared to the non-treated control (Figure 5A-C). In pPBMCs treated with ETEC K99 in the presence of EK99P-1, IL-1 $\beta$ and $I L-8$ levels were comparably low to control levels (Figure 5A, B). However, we observed no differences in the mRNA expression of IFN-ywhen cells were infected with ETEC K99 in the presence of EK99P-1 compared to ETEC K99 alone (Figure 5C). 
No significant change in the major immune cells composing pPBMCs (CD172a ${ }^{+}$myeloid cells; $\mathrm{CD}^{+}$total $\mathrm{T}$ cells; or $\mathrm{CD}^{+}, \mathrm{CD}^{+}$, or $\mathrm{CD} 4^{+} \mathrm{CD} 8^{+} \mathrm{T}$ cells) was observed following ETEC K99 infection and phage EK99P-1 treatment (Figure 5D). Together, these data suggest that phage EK99P-1 treatment prevents inflammatory responses caused by ETEC K99 infection by reducing inflammatory cytokines in the immune cells composing pPBMCs.

\section{Discussion}

In this study, we examined the effects of phage EK99P-1 on barrier protection and cytokine production in porcine IECs infected with ETEC in the presence or absence of immune cells. Upon ETEC infection, barrier disruption in the host is thought to occur due to the action of enterotoxins, such as LT or ST, secreted from the ETEC. Since enterotoxin secretion occurs upon the attachment of ETEC to host IECs, major barrier disruption is initiated by ETEC adhesion $[7,8]$. Therefore, the alleviation of barrier disruption by phage EK99P-1 treatment against ETEC K99 infection may be at least partially, if not entirely, caused by decreased ETEC K99 adhesion.

A disrupted barrier favors indiscriminate and easy penetration by pathogens, and impairment of normal physiological function and the local mucosal immune system, often causing an inflammatory reaction [24]. ETEC infection has been reported to induce increased serum IL-8, which was lowered by dietary phage supplementation in post-weaning pigs $[12,13]$. The current study revealed the induction of IL-8 in cells infected with ETEC K99 in both IECs and immune cells, and its reduction by phage EK99P-1 treatment.

Because IECs are in close contact with immune cells in the lamina propria of the intestinal tract, we hypothesized that the modulation of chemokine secretion by phage EK99P-1, in the context of IECs infected with ETEC K99, could influence basolateral immune cells. Therefore, we adopted the IPECJ2/PBMC co-culture system [23] and examined the effect of phage EK99P-1 on immune response modulation. After co-culture, $I L-1 \beta$ and $I L-8$ expression, upregulated by ETEC K99 infection, were diminished by phage EK99P-1 treatment in pPBMCs. One reason for the low levels of inflammatory cytokines in basolateral pPBMCs may be that soluble toxins, such as LT and ST, secreted from ETEC did not permeate the IEC barrier. Our results showed that both 4- and 40-kD dextran-FITC permeability was restored to control levels by phage EK99P-1 treatment against ETEC K99 exposure to IPEC-J2 cells. These results suggest that the basolateral translocation of soluble toxins, especially ST, may also be reduced due to phage EK99P-1 treatment. Indeed, previous reports using enterotoxindeficient ETEC mutants demonstrated that STb specifically regulates immune-related genes, such as IL17A, IL-1 a, and IL-1 $\beta$ [25]. In addition, ST enhances the expression of pro-inflammatory cytokines and chemokines, such as IL-6 and IL-8, in the small intestine [26]. However, further studies are needed to precisely determine the factors involved in inflammation modulation by immune cells.

Our results also showed that mRNA expression of IFN- $\gamma$, produced mainly by T cells [27], was not significantly different in PPBMCs co-cultured with IPEC-J2 cells infected with ETEC K99, with or without 
phage EK99P-1. Therefore, it is probable that the control of ETEC K99 by phage EK99P-1 is unrelated to IFN- $\gamma$ expression by $T$ cells. However, reduced mRNA expression of $I L-1 \beta$ and $i / 8$ was observed in a cocultured system of treated ETEC K99 and phage EK99P-1, compared to ETEC K99 alone. Considering that the main cells producing IL-1 $\beta$ and IL-8 in pPBMCs are myeloid cells [28], we infer a possible difference in cytokine production capacity, although no proportional change in the population of cells was observed. Our results show that phage EK99P-1 blocks intestinal barrier disruption and inhibits inflammatory responses in IPEC-J2 cells infected with ETEC K99, by reducing ETEC K99 adhesion. Reinforcement of the intestinal barrier by phage EK99P-1 also modulates the immune cell inflammatory response.

\section{Declarations}

\section{Acknowledgements}

We thank iNtRON Biotechnology, Seongnam, Korea for providing the phage EK99P-1 strain, and the National Instrumentation Center for Environmental Management (NICEM) at Seoul National University, Korea for technical support. This work was conducted with the support of the Cooperative Research Program for Agriculture Science and Technology Development (project no. PJ016201), Rural Development Administration, Republic of Korea. This research was also funded by the Korea Institute of Planning and Evaluation for Technology in Food, Agriculture, Forestry, and Fisheries (IPET 319081-03). DBJ and YJP were supported by the BK21 FOUR Program of the Department of Agricultural Biotechnology, Seoul National University, Seoul, Republic of Korea.

\section{Authors' contributions}

C-HY conceived the study; SHH, B-CP, and C-HY designed the study; NK, MJG, Y-CK, Y-JJ, RH, DBJ, and YJP performed the experiments and analyzed the data. NK and C-HY wrote the manuscript. All authors reviewed, discussed, and agreed to submit the manuscript.

\section{Competing interests}

The authors declare no competing interests.

\section{Ethics dclarations}

The porcine blood samples used in this study were approved by the Institutional Animal Care and Use Committee of Seoul National University (IACUC no. SNU-150327-2).

\section{References}

1. Wilson, R. and D.J.A.j.o.v.r. Francis, Fimbriae and enterotoxins associated with Escherichia coli serogroups isolated from pigs with colibacillosis. 1986. 47(2): p. 213-217.

2. Kaper, J.B., J.P. Nataro, and H.L.J.N.r.m. Mobley, Pathogenic escherichia coli. 2004. 2(2): p. 123-140. 
3. Nagy, B. and P.Z. Fekete, Enterotoxigenic Escherichia coli (ETEC) in farm animals. Veterinary research, 1999. 30(2-3): p. 259-284.

4. Chen, X., et al., Prevalence of serogroups and virulence factors of Escherichia coli strains isolated from pigs with postweaning diarrhoea in eastern China. Veterinary microbiology, 2004. 103(1-2): p. 13-20.

5. Shams, Z., et al., Detection of enterotoxigenic K99 (F5) and F41 from fecal sample of calves by molecular and serological methods. Comparative clinical pathology, 2012. 21(4): p. 475-478.

6. Xia, P., et al., Deletion of FaeG alleviated Enterotoxigenic Escherichia coli F4ac-induced apoptosis in the intestine. 2021. 11(1): p. 1-8.

7. Nagy, B. and P.Z. Fekete, Enterotoxigenic Escherichia coli in veterinary medicine. International Journal of Medical Microbiology, 2005. 295(6-7): p. 443-454.

8. Croxen, M.A. and B.B.J.N.R.M. Finlay, Molecular mechanisms of Escherichia coli pathogenicity. 2010. 8(1): p. 26-38.

9. Luo, Y., et al., Toll-like receptor 5-mediated IL-17C expression in intestinal epithelial cells enhances epithelial host defense against F4+ ETEC infection. 2019. 50(1): p. 1-14.

10. Zanello, G., et al., Saccharomyces cerevisiae modulates immune gene expressions and inhibits ETECmediated ERK1/2 and p38 signaling pathways in intestinal epithelial cells. 2011. 6(4): p. e18573.

11. Karthik, L., et al., Protease inhibitors from marine actinobacteria as a potential source for antimalarial compound. 2014. 9(3): p. e90972.

12. Lee, C., et al., Effects of dietary supplementation of bacteriophages against enterotoxigenic Escherichia coli (ETEC) K88 on clinical symptoms of post-weaning pigs challenged with the ETEC pathogen. Journal of animal physiology and animal nutrition, 2017. 101(1): p. 88-95.

13. Heo, J., et al., Gastrointestinal health and function in weaned pigs: a review of feeding strategies to control post-weaning diarrhoea without using in-feed antimicrobial compounds. Journal of animal physiology and animal nutrition, 2013. 97(2): p. 207-237.

14. Cheng, G., et al., Antibiotic alternatives: the substitution of antibiotics in animal husbandry? Frontiers in microbiology, 2014. 5: p. 217.

15. Wittebole, X., S. De Roock, and S.M. Opal, A historical overview of bacteriophage therapy as an alternative to antibiotics for the treatment of bacterial pathogens. Virulence, 2014. 5(1): p. 226-235.

16. Smith, H.W. and M. Huggins, Effectiveness of phages in treating experimental Escherichia coli diarrhoea in calves, piglets and lambs. Microbiology, 1983. 129(8): p. 2659-2675. 
17. Jamalludeen, N., et al., Evaluation of bacteriophages for prevention and treatment of diarrhea due to experimental enterotoxigenic Escherichia coli 0149 infection of pigs. Veterinary microbiology, 2009. 136(1-2): p. 135-141.

18. Cha, S.B., et al., Effect of bacteriophage in enterotoxigenic Escherichia coli (ETEC) infected pigs. Journal of Veterinary Medical Science, 2012. 74(8): p. 1037-1039.

19. Gu, M.J., et al., Bacillus subtilis protects porcine intestinal barrier from deoxynivalenol via improved zonula occludens-1 expression. 2014. 27(4): p. 580.

20. Sargeant, H.R., H.M. Miller, and M.-A.J.M.i. Shaw, Inflammatory response of porcine epithelial IPEC J2 cells to enterotoxigenic E. coli infection is modulated by zinc supplementation. 2011. 48(15-16): p. 21132121.

21. Larsen, S.B., C.J. Cowley, and E.J.C.o.i.i. Fuchs, Epithelial cells: liaisons of immunity. 2020. 62: p. 4553.

22. Peterson, L.W. and D. Artis, Intestinal epithelial cells: regulators of barrier function and immune homeostasis. Nature Reviews Immunology, 2014. 14(3): p. 141.

23. Gu, M.J., et al., Barrier protection via Toll-like receptor 2 signaling in porcine intestinal epithelial cells damaged by deoxynivalnol. 2016. 47(1): p. 1-11.

24. Roselli, M., et al., The novel porcine Lactobacillus sobrius strain protects intestinal cells from enterotoxigenic Escherichia coli K88 infection and prevents membrane barrier damage. 2007. 137(12): p. 2709-2716.

25. Loos, M., et al., Role of heat-stable enterotoxins in the induction of early immune responses in piglets after infection with enterotoxigenic Escherichia coli. 2012. 7(7): p. e41041.

26. Loos, M., et al., Optimization of a small intestinal segment perfusion model for heat-stable enterotoxin A induced secretion in pigs. 2013. 152(1-2): p. 82-86.

27. Cheon, I.S., et al., Functional characteristics of porcine peripheral T cells stimulated with IL-2 or IL-2 and PMA. 2014. 96(1): p. 54-61.

28. Cassatella, M.A., et al., Interleukin 10 (IL-10) inhibits the release of proinflammatory cytokines from human polymorphonuclear leukocytes. Evidence for an autocrine role of tumor necrosis factor and IL-1 beta in mediating the production of IL-8 triggered by lipopolysaccharide. 1993. 178(6): p. 2207-2211.

29. Seo, B.-J., et al., Evaluation of the broad-spectrum lytic capability of bacteriophage cocktails against various Salmonella serovars and their effects on weaned pigs infected with Salmonella Typhimurium. 2018: p. 17-0501. 
30. Gu, M.J., et al., Hydrolyzed fumonisin B1 induces less inflammatory responses than fumonisin B1 in the co-culture model of porcine intestinal epithelial and immune cells. Toxicol Lett, 2019. 305: p. 110-116.

\section{Tables}

Due to technical limitations, table 1 is only available as a download in the Supplemental Files section.

\section{Figures}

A
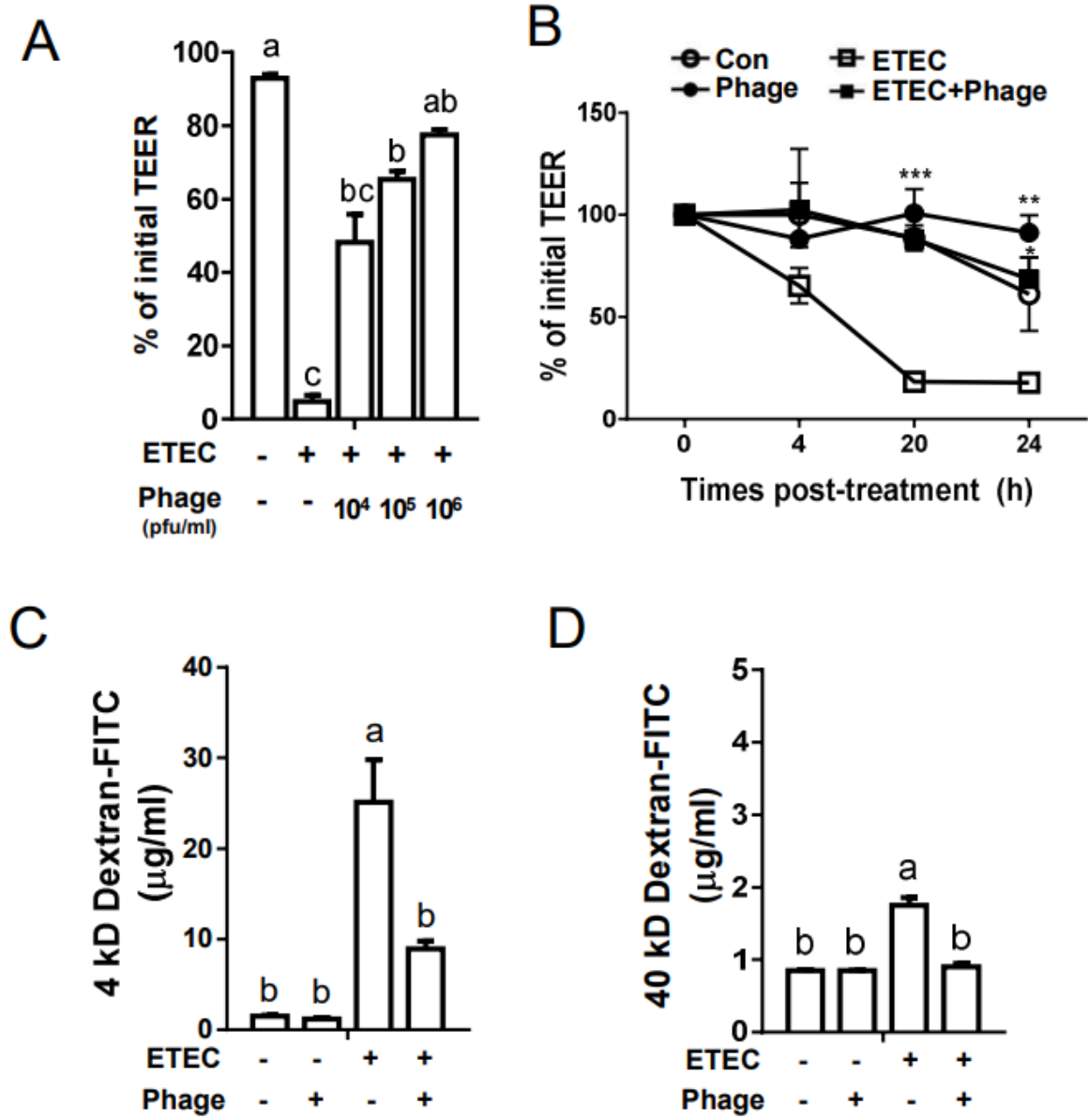

\section{Figure 1}

Phage EK99P-1 restored intestinal permeability in porcine intestinal epithelial cell line IPEC-J2 cells infected with enterotoxigenic Escherichia coli (ETEC K99). Differentiated IPEC-J2 cells were treated with ETEC K99 $(1 \times 107 \mathrm{cfu} / \mathrm{mL})$ and phage EK99P-1 $(1 \times 106 \mathrm{pfu} / \mathrm{mL})$ for $24 \mathrm{~h}$. (A) Transepithelial electrical resistance (TEER) was measured in epithelial volt/ohm after $24 \mathrm{~h}$ of infection and (B) at the indicated time points. Each datum represents a percentage of initial TEER $(n=3)$. ${ }^{\star *} P<0.01$; ${ }^{\star \star *} P<0.001$. (C, D) Permeability was assessed by measuring 4- or 40-kD fluorescein isothiocyanate (FITC)-dextran transport after $24 \mathrm{~h}$ of infection $(\mathrm{n}=3)$. Means were compared by one-way analysis of variance (ANOVA), followed by the Friedman test and Tukey's multiple comparison test. Data are expressed as means \pm standard deviation (SD). Different letters in each group indicate significant differences at $\mathrm{P}<0.05$. 


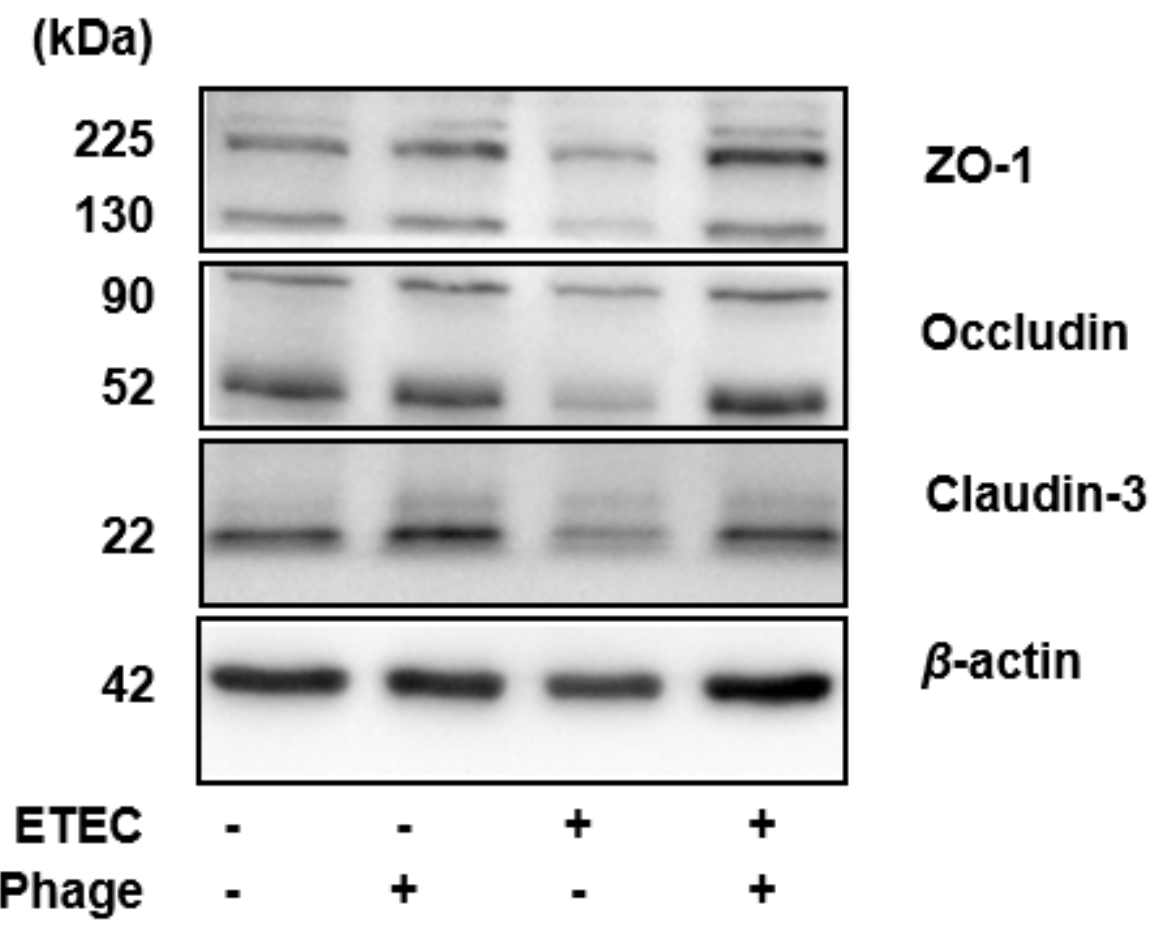

Figure 2

Phage EK99P-1 restored the decreased expression of tight junction proteins by ETEC in IPEC-J2 cells infected with ETEC K99. A monolayer of confluent IPEC-J2 cells was treated with ETEC K99 $(2 \times 106$ $\mathrm{cfu} / \mathrm{mL})$ and phage EK99P-1 $(2 \times 105 \mathrm{pfu} / \mathrm{mL})$ for $3 \mathrm{~h}$. Whole-cell lysates were analyzed for the expression of ZO-1, occludin, claudin-3, and $\beta$-actin using anti- claudin-3, -occludin, ZO-1 and $\beta$-actin antibodies by western blot assay $(n=3)$.

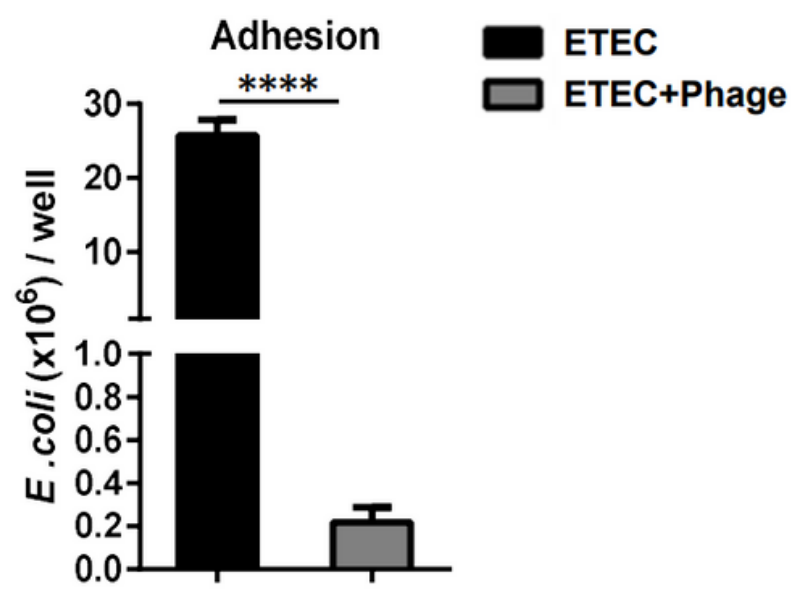

Figure 3

Phage EK99P-1 inhibited the adhesion of ETEC K99 to IPEC-J2 cells. Differentiated IPEC-J2 cells were treated with ETEC K99 $(1 \times 107 \mathrm{cfu} / \mathrm{mL})$ and phage EK99P-1 $(1 \times 106 \mathrm{pfu} / \mathrm{mL})$ for $24 \mathrm{~h}$. An E. coli 
adhesion assay was performed on IPEC-J2 cells $(n=3)$. Means were compared using a two-tailed Student's t-test. Data are means \pm SD. ${ }^{\star \star \star *} \mathrm{P}<0.0001$ compared to the ETEC control.

A

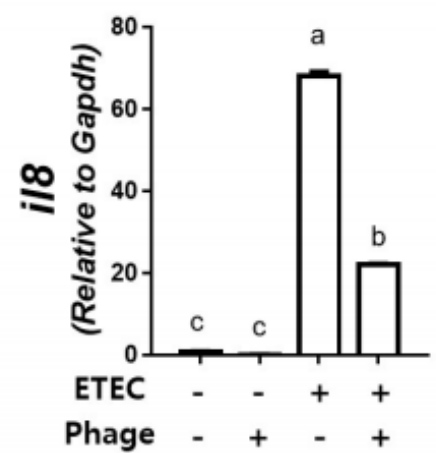

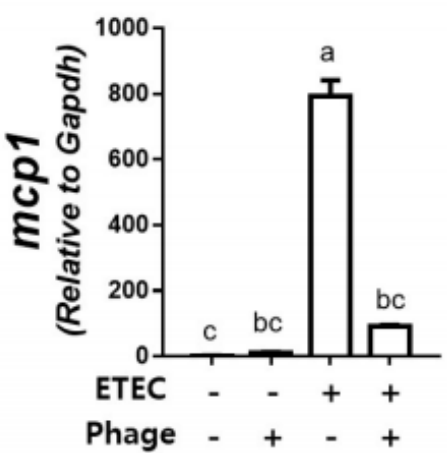

B

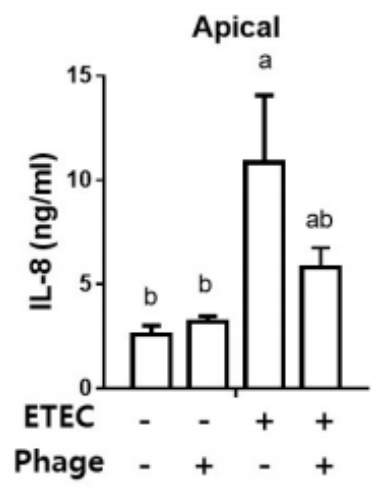

Basolateral

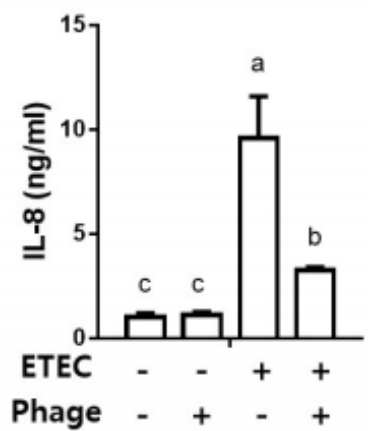

\section{Figure 4}

Phage EK99P-1 inhibits inflammatory responses in IPEC-J2 infected with ETEC K99. Differentiated IPECJ2 cells were treated with ETEC K99 $(1 \times 107 \mathrm{cfu} / \mathrm{mL})$ and phage EK99P- $1(1 \times 106 \mathrm{pfu} / \mathrm{mL})$ for $24 \mathrm{~h} .(\mathrm{A})$ The expression of cytokine and chemokine mRNA in differentiated IPEC-J2 cells was measured by reverse-transcription polymerase chain reaction (RT-PCR) after $24 \mathrm{~h}$ of treatment $(n=3)$. (B) Interleukin (IL)-8 secretion on the apical or basolateral side of differentiated IPEC-J2 cells was measured by an enzyme-linked immunosorbent assay (ELISA; $n=3)$. Means were compared using one-way ANOVA, followed by Friedman's test and Tukey's multiple comparison test. Data are means \pm SD. Different letters in each group indicate significant differences at $\mathrm{P}<0.05$. 

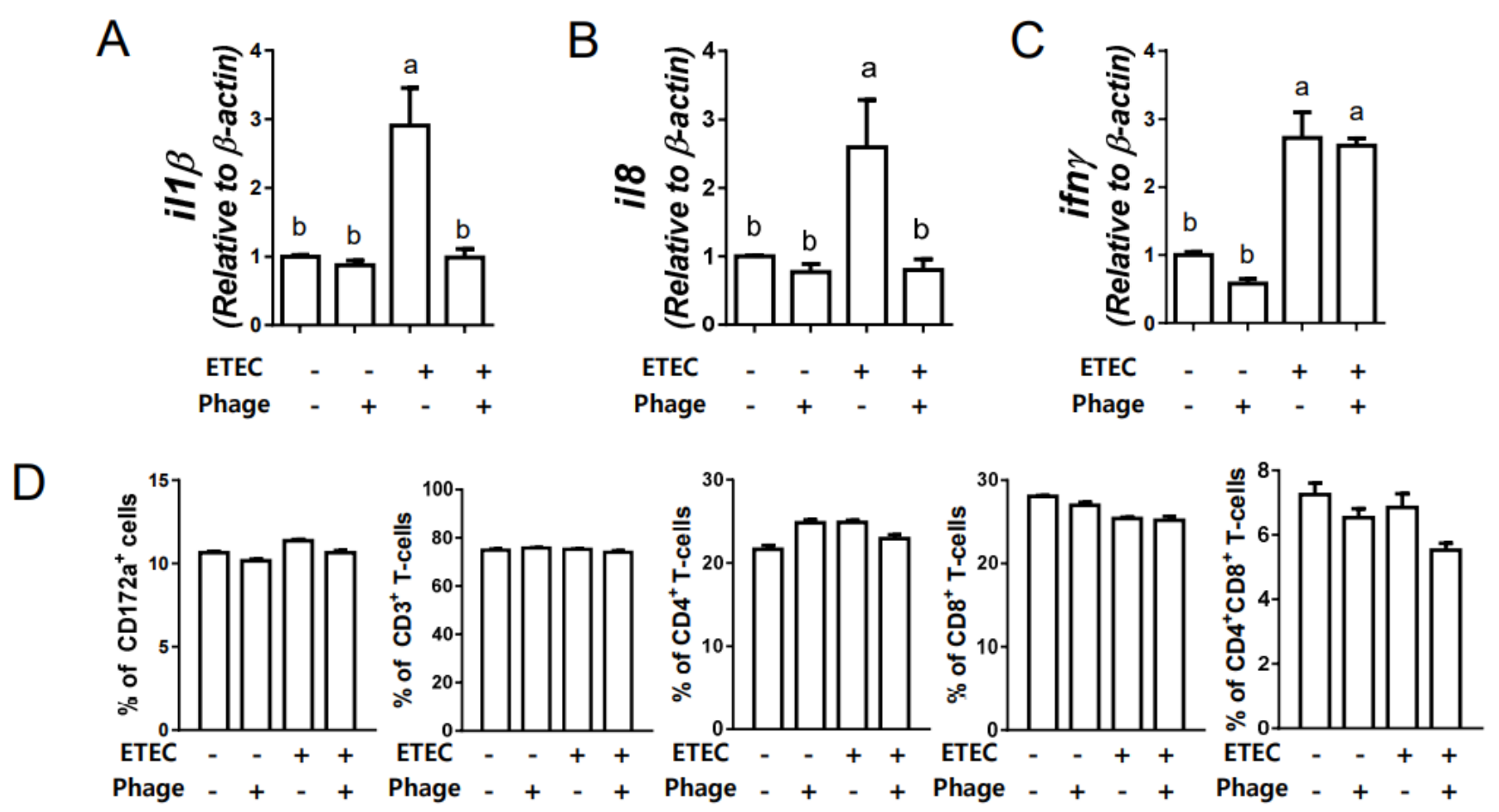

Figure 5

Phage EK99P-1 alleviated the inflammatory response of immune cells under IPEC-J2 infection with ETEC K99. Differentiated IPEC-J2 cells and pPBMCs were co-cultured using Transwell plates. IPEC-J2 cells were treated with ETEC K99 $(1 \times 107 \mathrm{cfu} / \mathrm{mL})$ and phage EK99P-1 $(1 \times 106 \mathrm{pfu} / \mathrm{mL})$ on the apical side for $24 \mathrm{~h}$. (A-C) Expression of cytokine mRNA in pPBMC was measured by real-time quantitative RT-PCR (qRT-PCR; $\mathrm{n}$ = 3). (D) pPBMCs were stained with anti-CD172a, - CD3e, - CD4, or $-C D 8 a$ antibodies to analyze the population of immune cells using flow cytometry. Data are mean percentages of each cell population relative to the total cells $\pm S D(n=3)$. Means were compared using one-way ANOVA, followed by a Friedman test corrected by Tukey's multiple comparison test. Data are means \pm SD. Different letters in each group indicate significant differences at $\mathrm{P}<0.05$.

\section{Supplementary Files}

This is a list of supplementary files associated with this preprint. Click to download.

- Supplementaryfile2.pdf

- table1.xlsx 